\title{
Direkte Diskriminierung der Männer in der Bundesgesetzgebung
}

\author{
Marco Frei *
}

Der Beitrag zeigt, dass Männer gegenüber Frauen in diversen Bundesgesetzen direkt benachteiligt werden. Die Benachteiligungen lassen sich meist durch die traditionelle Rollenverteilung zwischen Mann und Frau erklären, jedoch nicht durch biologisch-funktionale Geschlechterunterschiede rechtfertigen. Aufgrund der eingeschränkten Verfassungsgerichtsbarkeit gegenüber Bundesgesetzen (Art. 19o BV) ist es grundsätzlich Sache des Gesetzgebers, das Bundesrecht grundrechtskonform auszugestalten (Art. 35 Abs. 1 BV). Lösungsansätze für die geschlechtergerechte Ausgestaltung des Bundesrechts werden im Beitrag angedeutet.

I. Einleitung .46

II. Die überwundene Diskriminierung im ehelichen Namensrecht .......................47

III. Diskriminierung im geltenden Recht .......................................................49

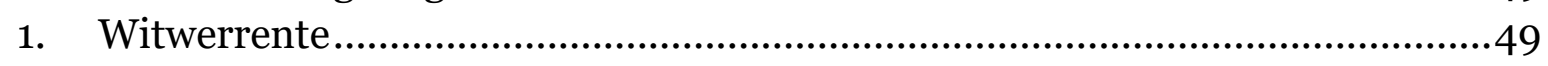

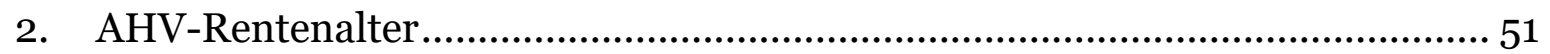

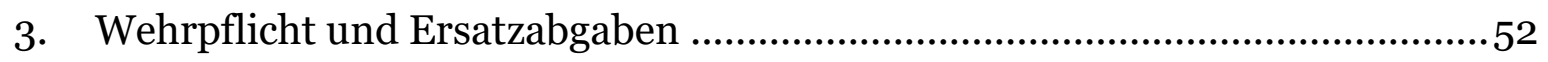

IV. Ein Grenzfall: Der Vergewaltigungstatbestand (Art. 190 StGB) ...........................54

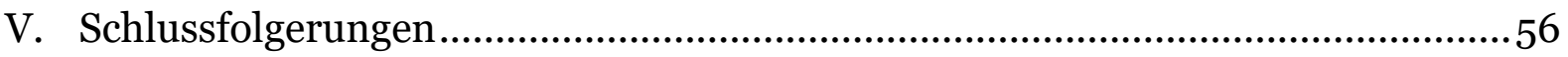

Zitiervorschlag: Marco Frei, Direkte Diskriminierung der Männer in der Bundesgesetzgebung, in: sui-generis 2020, S. 45

URL: $\quad$ sui-generis.ch/121

DOI: $\quad$ https://doi.org/10.21257/sg.121

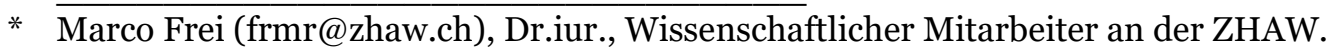




\section{Einleitung}

1 Gemäss Art. 8 Abs. 3 BV sind «Mann und Frau (...) gleichberechtigt. Das Gesetz sorgt für ihre rechtliche und tatsächliche Gleichstellung, vor allem in Familie, Ausbildung und Arbeit. Mann und Frau haben Anspruch auf gleichen Lohn für gleichwertige Arbeit.» Hierbei handelt sich um die Nachfolgebestimmung des Art. 4 Abs. 2 aBV. Dieser wurde als direkter Gegenvorschlag zu einer im «Internationalen Jahr der Frau» (1975) lancierten Verfassungsinitiative zur Verbesserung der Stellung der Frau in die Verfassung aufgenommen. ${ }^{1}$ Der Gegenvorschlag war derselben Idee verpflichtet. ${ }^{2}$ Gleichwohl geht das Bundesgericht davon aus, dass Art. 8 Abs. 3 BV die Gleichstellung der Geschlechter bezweckt: Die Bestimmung «verbietet nicht nur die Benachteiligung von Frauen, sondern jede nicht durch zwingende biologische oder funktionale Unterschiede gerechtfertigte geschlechtsbezogene Regelung, unabhängig davon, ob dadurch Frauen oder Männer benachteiligt werden; das Recht muss geschlechtsneutral sein».3

\footnotetext{
Botschaft über die Volksinitiative «Gleiche Rechte für Mann und Frau» vom 14. November 1979, BBl 1980 I S. 69 ff.; Bericht des Bundesrates über das Rechtsetzungsprogramm «Gleiche Rechte für Mann und Frau» vom 26. Februar 1986 (86.008); Bundesratsbeschluss über das Ergebnis der Volksabstimmung vom 14. Juni 1981 (Gleiche Rechte für Mann und Frau; Konsumentenschutz) vom 17. August 1981, BBl 1981 II S. 1266 ff. Damit war die Diskussion darum beendet, ob sich der Grundsatz der Gleichbehandlung der Geschlechter aus dem allgemeinen Rechtgleichheitsgebot ableiten lässt: Margrith Bigler-Eggenberger/ Regula Kägi-Diener, N 88 zu Art. 8 BV, in: Bernhard Ehrenzeller/Benjamin Schindler/Rainer J. Schweizer/Klaus A. Vallender (Hrsg.), Die schweizerische Bundesverfassung, St. Galler Kommentar, 3. Aufl. 2014.

2 SGK BV-Bigler-Eggenberger/Kägi-Diener (Fn. 1), Art. 8 N 101.

3 BGE 129 I 265 E. 3.3; vgl. auch BGE 126 I 1 E. 2b/c mit Hinweisen. Auf den Gleichstellungsartikel
}

2 Die «Geschlechtsneutralität des Rechts» ist ein Ideal. Die Bundesgesetzgebung hat sich diesem in gewissen Bereichen angenähert. Beispielsweise ist das eheliche Namensrecht heute geschlechtsneutral ausgestaltet (Art. 160 ZGB). In diversen Bestimmungen der Bundesgesetzgebung wird das Geschlecht jedoch noch heute als Kriterium für eine Ungleichbehandlung herangezogen. Beispielhaft erwähnt seien die Militärdienstpflicht (Art. $2 \mathrm{ff}$. MG), die Regelungen über die Anspruchsberechtigung für die AHV-Rente (Art. 21 AHVG) sowie die Witwen- und Witwerrenten (Art. 23 f. AHVG) und der Vergewaltigungstatbestand (Art. $190 \mathrm{StGB}$ ). 4

3 Eine direkte Geschlechterdiskriminierung liegt vor, «wenn sich eine Ungleichbehandlung ausdrücklich auf die Geschlechtszugehörigkeit oder auf ein Kriterium stützt, das nur von einem der beiden Geschlechter erfüllt werden kann, und wenn sie sich nicht sachlich rechtfer-

haben sich nach der Annahme zunächst vorwiegend Männer berufen: vgl. z.B. BGE $106 \mathrm{Ib}$ 182, BGE $109 \mathrm{Ib} 81$; BGE 116 V 198; BGE 117 V 318; BGE 117 V 194 E. 4， 196; BGE 119 V 277; BGE 120 V 312; BGE 121 V 229; BGE 123 V 189; BGE 123 I 56.

4 Auch die geplante Quotenregelung im Aktienrecht (Art. $734 f$ E-OR) ist geschlechtsbezogen, die beiden Geschlechter werden indessen formal gleichbehandelt: Entwurf des Obligationenrechts (Aktienrecht) vom 23. November 2016 (BBl 2017 683) S. 725. Art. $734 f$ E-OR lautet: «Sofern nicht jedes Geschlecht mindestens zu 30 Prozent im Verwaltungsrat und zu 20 Prozent in der Geschäftsleitung vertreten ist, sind im Vergütungsbericht bei Gesellschaften, welche die Schwellenwerte gemäss Artikel 727 Absatz 1 Ziffer 2 überschreiten, anzugeben: 1. die Gründe, weshalb die Geschlechter nicht wie vorgesehen vertreten sind; und 2. die Massnahmen zur Förderung des weniger stark vertretenen Geschlechts.» Zur Vereinbarkeit der Quotenregelung mit der Bundesverfassung, insbesondere der Wirtschaftsfreiheit der betroffenen Unternehmen, vgl. Jakob Ueberschlag, Geschlechtsspezifische Quotenregelung im Rahmen der geplanten Aktienrechtsrevision, AJP 2015, S. 907 ff. 
tigen lässt». 5 In den vorerwähnten Bestimmungen der Bundesgesetzgebung waren oder sind Männer gegenüber Frauen benachteiligt. Nachfolgend wird darauf eingegangen, ob die Ungleichbehandlung durch zwingende biologische Unterschiede gerechtfertigt ist oder einer solchen Rechtfertigung entbehrt.

\section{Die überwundene Diskriminierung im ehelichen Namensrecht}

4 Die deutsch-schweizerische Doppelbürgerin Susanna Burghartz und der Schweizer Bürger Albert Schnyder, beide seit 1975 in Basel wohnhaft, heirateten 1984 in Deutschland. Sie bestimmten den Namen Burghartz nach deutschem Recht als Familiennamen und der Bräutigam machte von seinem Recht Gebrauch, seinen bisherigen Namen voranzustellen. Er führte somit den Namen «Schnyder Burghartz». Die Schweizer Behörden trugen den Namen «Schnyder» als gemeinsamen Familiennamen

\footnotetext{
BGE 124 II 409 E. 7 und BGE 124 II 529 E. 3a; siehe auch Botschaft vom 24. Februar 1993 zum Bundesgesetz über die Gleichstellung von Frau und Mann (Gleichstellungsgesetz) und zum Bundesbeschluss über die Genehmigung einer Änderung der Verordnung über die Zuweisung der Ämter an die Departemente und der Dienste an die Bundeskanzlei (BBl 1993 I 1248), S. 1295. Das Gegenstück der direkten Geschlechterdiskriminierung bildet die indirekte Geschlechterdiskriminierung: vgl. dazu Jakob Ueberschlag, Anforderungen an das Vorliegen einer stärkeren nachteiligen Betroffenheit bei der indirekten Geschlechterdiskriminierung, AJP 2014 S. $361 \mathrm{ff}$. Diskriminierungen aufgrund der sexuellen Orientierung sind grundsätzlich keine Geschlechterdiskriminierungen, es sei denn, sie seien geeignet, ausschliesslich oder überwiegend die Angehörigen eines bestimmten Geschlechts zu benachteiligen, etwa wenn die Arbeitgeberschaft zwar gewillt ist, homosexuelle Frauen anzustellen, nicht aber homosexuelle Männer oder umgekehrt: BGE 145 II 153 E. 4.5; kritisch Sandra Hotz, Auf dem Weg zur Gleichstellung der Geschlechter?, SJZ 2020, S. 3 ff., S. 10 f.
}

der Ehegatten im Zivilstandsregister ein. Deshalb stellten die Ehegatten ein Namensänderungsgesuch mit den Anträgen, es sei ihnen die Führung des Namens «Burghartz» als Familienname und jene des Namens «Schnyder Burghartz» als Name des Ehegatten zu gestatten. Das Gesuch wurde am 6. November 1984 abgewiesen. ${ }^{6}$

5 In der zweiten Hälfte der 1980er-Jahre wurden die Bestimmungen über die Wirkungen der Ehe revidiert. Art. 160 Abs. 1 ZGB sah vor, dass der Name des Ehemannes der Familienname der Ehegatten ist. Abs. 2 dieses Artikels hielt fest, dass die Braut ihren bisherigen Namen dem Familiennamen voranstellen kann. Art. 30 ZGB («Namensänderung») legte in Abs. 2 zudem fest, dass «das Gesuch der Brautleute, von der Trauung an den Namen der Braut als Familiennamen zu führen, [...] zu bewilligen [ist], wenn achtenswerte Gründe vorliegen». Das seit dem 1. Januar 1988 in Kraft stehende Namensrecht gestattete es dem Ehemann nicht, seinen bisherigen Namen dem Namen der Frau voranzustellen, wenn dieser gemäss Art. 30 Abs. 2 ZGB von den Brautleuten als Familienname gewählt wurde. 7 Art. 30 Abs. 1 ZGB war von der Revision nicht betroffen. Die Bestimmung lautete unverändert wie folgt: «Die Regierung des Wohnsitzkantons kann einer Person die Änderung des Namens bewilligen, wenn wichtige Gründe vorliegen.»8

6 BGE 115 II 193 S. 194.

7 Botschaft des Bundesrates vom 11. Juli 1979 über die Änderung des Schweizerischen Zivilgesetzbuches (Wirkungen der Ehe im allgemeinen, Ehegüterrecht und Erbrecht), BBl 1979 II 1191 ff.; Änderung des Zivilgesetzbuches vom 5. Oktober 1984, AS 1986122.

8 Fassung gemäss Ziff. I 2 des BG vom 25. Juni 1976, in Kraft seit 1. Januar 1978 (AS 1977 237, 
Nach dem Inkrafttreten der neuen Regelungen zu den Wirkungen der Ehe stellten die Ehegatten erneut ein Gesuch. Die kantonalen Behörden lehnten auch dieses Gesuch ab und begründeten dies damit, dass den Eheleuten kein ernsthafter Nachteil aus der Führung des Familiennamens «Schnyder» erwachse (vgl. Art. 30 Abs. 1 ZGB). Der neue Art. 30 Abs. 2 ZGB werde zudem nicht rückwirkend auf Ehepaare angewendet, deren Ehe vor dem 1. Januar 1988 geschlossen worden sei, und der neue Art. 160 Abs. 2 ZGB gelte nur für die Ehegattin. 9

7 Das Bundesgericht hiess die Berufung der Ehegatten teilweise gut: Die Anwendung von Art. 30 Abs. 2 ZGB komme zwar nicht infrage, doch lägen in diesem Fall wichtige Gründe für die Anwendung des Abs. 1 dieses Artikels vor, insbesondere das Alter und das berufliche Wirkungsfeld der Eheleute sowie die Unterschiede zwischen Deutschland und der Schweiz in der einschlägigen Gesetzgebung, welche durch die Nähe der Grenzstadt Basel zu Deutschland noch stärker ins Gewicht fallen. Deshalb soll den Ehegatten erlaubt werden, Burghartz als Familiennamen zu führen. Das Bundesgericht lehnte hingegen das Gesuch des Ehemannes ab, den Namen «Schnyder Burghartz» tragen $\mathrm{zu}$ dürfen. Das Schweizer Parlament, das an der Einheit des Namens in der Familie festhalten und einen radikalen Bruch mit der Tradition vermeiden wolle, habe sich nie für

264; Botschaft des Bundesrates an die Bundesversammlung vom 5. Juni 1974 über die Änderung des Schweizerischen Zivilgesetzbuches [Kindesverhältnis], BBl 1974 II 1 ff.).

9 Bericht der Kommission für Rechtsfragen des Nationalrates vom 27. August 2009 über die parlamentarische Initiative «Name und Bürgerrecht der Ehegatten. Gleichstellung» (BBl 2009 7573) S. 7576 . die absolute Gleichstellung der Ehegatten bei der Namenswahl ausgesprochen und somit bewusst nur der Frau erlaubt, ihren bisherigen Namen dem Familiennamen voranzustellen. ${ }^{10}$

8 Danach hatten die Europäische Kommission für Menschenrechte und schliesslich der Europäische Gerichtshof für Menschenrechte über die Beschwerde der Eheleute zu befinden. Letzterer verkündete sein Urteil am 22. Februar 1994. Er hatte den Fall in Bezug auf die Art. 8 (Recht auf Achtung des Privat- und Familienlebens) und Art. 14 (Diskriminierungsverbot) der EMRK geprüft und dabei dem Vorbehalt betreffend Namensrecht, den die Schweiz im Zusammenhang mit dem Protokoll Nr. 7 angebracht hatte, die Wirkung versagt. Art. 5 dieses Protokolls («Gleichberechtigung der Ehegatten») sieht vor, dass die Ehegatten «hinsichtlich der Eheschliessung, während der Ehe und bei Auflösung der Ehe [...] untereinander und in ihren Beziehungen zu den Kindern gleiche Rechte und Pflichten privatrechtlicher Art» haben. Der Gerichtshof erinnerte daran, dass die Entwicklung hin zur vollständigen Gleichstellung der Geschlechter eines der Hauptziele der Mitgliedstaaten des Europarates ist und deshalb eine einzig durch das Geschlecht begründete Ungleichbehandlung nur aus überaus gewichtigen Gründen als mit der Konvention vereinbar erachtet werden kann. Da in den Augen des Gerichtshofs keine objektiven und vernünftigen Gründe für die Ungleichbehandlung der Ehegatten ersichtlich sind, kam er zum Schluss, dass

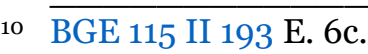


eine Verletzung der Art. 8 und 14 EMRK vorliegt. ${ }^{11}$

9 Aufgrund des Urteils des EGMR änderte der Bundesrat die Zivilstandsverordnung per 1. Juli 1994. Dem Mann wurde gestattet, seinen Namen dem Familiennamen voranzustellen, wenn die Brautleute beantragt haben, den Namen der Frau als Familiennamen zu führen. Im Rahmen der Totalrevision der Zivilstandsverordnung wurde diese Bestimmung zu Art. 12 Abs. 1 zweiter Satz. ${ }^{12}$ Das ZGB stand nun im Widerspruch zur Verordnung und zum Völkerrecht. Schliesslich wurde der Widerspruch behoben. Die Bundesversammlung beschloss nach Einsicht in den Bericht der Kommission für Rechtsfragen des Nationalrates vom 27. August $2009^{13}$ und in die Stellungnahme des Bundesrates vom 14. Oktober 200914, das ZGB so anzupassen, dass Doppelnamen für Ehefrauen und Ehemänner möglich sind.15 Das Namensrecht wurde später

11 Urteil des EGMR 16213/90 vom 22. Februar 1994 (Burghartz vs. Schweiz), Serie A Nr. 280. Eine vertiefte Sicht auf das Verbot der direkten Diskriminierung im Anwendungsbereich der EMRK bietet Tilmann Altwicker, Menschenrechtlicher Gleichheitsschutz, Heidelberg 2011, S. 255 ff.

12 Zivilstandsverordnung vom 28. April 2004 (ZStV; SR 211.112.2).

13 BBl 20097575 ff. (Fn. 9).

14 Stellungnahme des Bundesrates vom 14. Oktober 2009 zum Bericht der Kommission für Rechtsfragen des Nationalrates vom 27. August 2009 über die parlamentarische Initiative «Name und Bürgerrecht der Ehegatten. Gleichstellung», BBl $20097581 \mathrm{ff}$.

15 Die Abs. 2 und 3 des Art. 16o ZGB wurden wie folgt geändert (Schweizerisches Zivilgesetzbuch [Name und Bürgerrecht], BBl 2009 7579): «Die Braut kann jedoch gegenüber dem Zivilstandsbeamten oder der Zivilstandsbeamtin erklären, sie wolle ihren bisherigen Namen dem Familiennamen voranstellen. Die gleiche Möglichkeit hat der Bräutigam, wenn die Brautleute das Gesuch stellen, von der Trauung an den Namen der Ehefrau als Familiennamen zu führen (Art. 30 Abs. 2). Ist der vor der Trauung getragene Name bereits ein solcher Doppelname, so nochmals revidiert. Art. 160 ZGB in der aktuellen Fassung erlaubt Doppelnamen nicht mehr. ${ }^{16}$

10 Im Sinne eines Zwischenfazits kann festgehalten werden, dass die Geschlechterdiskriminierung im Namensrecht behoben ist. Bezüglich Doppelnamen sind heute beide Geschlechter - pointiert gesagt - gleich schlecht gestellt. Nicht zuletzt deshalb ist im Namensrecht keine Ruhe eingekehrt. Am 15. Dezember 2017 wurde im Nationalrat eine parlamentarische Initiative eingereicht: Heiratswilligen sei durch eine entsprechende Gesetzesänderung zu ermöglichen, nach der Eheschliessung künftig auch Doppelnamen tragen zu dürfen. Die Rechtskommission des Nationalrates hat der Initiative am 14. Januar 2019 Folge gegeben. ${ }^{17}$

\section{Diskriminierung im geltenden Recht}

\section{Witwerrente}

11 Rita K. stand als Primarlehrerin im Dienst des Kantons St. Gallen und war als solche seit anfangs Mai 1971 bei der Kantonalen Lehrerversicherungskasse (KLVK) versichert. Während ihrer Mitgliedschaft hatte sie Beiträge in der Höhe von Fr. 35'842.70 geleistet. Rita K. verstarb am 1. Februar 1986 und hinterliess als einzigen Erben ihren Ehemann,

kann lediglich der erste Name vorangestellt werden.»

16 Fassung gemäss Ziff. I des BG vom 30. Sept. 2011 (Name und Bürgerrecht), in Kraft seit 1. Jan. 2013 (AS 2012 2569; BBl 20097573 ff. [Fn. 9], S. $7581 \mathrm{ff}$. [Fn. 14]). Gleichzeitig wurde Art. 30 Abs. 1 ZGB noch angepasst: «Die Regierung des Wohnsitzkantons kann einer Person die Änderung des Namens bewilligen, wenn achtenswerte Gründe vorliegen.» Art. 30 Abs. 2 ZGB wurde gestrichen.

17 Parlamentarische Initiative Luzi Stamm (17.523). 
Heinz K. Am 2. April 1986 ersuchte Heinz K. die KLVK um Zusprechung einer Abfindung in der Höhe der Austrittsentschädigung (Freizügigkeitsleistung), die er auf Fr. 49'312.30 bezifferte. Er machte geltend, von seiner Ehefrau während der Ehedauer namhaft unterstützt worden zu sein, sei doch aus ihrem Einkommen der wesentliche Teil des gemeinsamen Lebensunterhalts bestritten, sein eigenes Einkommen hingegen vor allem für Anschaffungen verwendet worden. Nachdem die KLVK die wirtschaftlichen Verhältnisse durch Beizug verschiedener Unterlagen abgeklärt hatte, wies sie mit Verfügung vom 21. April 1986 das Gesuch um Abfindung ab, weil das in Art. 47 Abs. 1 der Verordnung über die kantonale Lehrerversicherungskasse vom 21. Januar 1964 (KLVK-V; sGS 213.55) aufgestellte Erfordernis der namhaften Unterstützung des hinterlassenen Ehemannes durch die verstorbene Ehefrau nicht erfüllt sei. Heinz K. beschwerte sich erfolglos bei der Erziehungsdirektion und dem kantonalen Versicherungsgericht. Das Bundesgericht hiess seine Beschwerde dagegen gut. Es stellte in BGE 116 V 198 fest: «Eine kantonalrechtliche Ordnung, wonach einerseits der Anspruch auf Witwerrente nur besteht, wenn der Witwer während der Ehe auf den Verdienst der Ehefrau angewiesen war und er nachher nicht voll erwerbsfähig ist, währenddem anderseits der Anspruch auf Witwenrente allein durch den Tod des Ehemannes begründet wird, stellt eine geschlechtsspezifische Unterscheidung dar, die sich weder mit biologischen noch mit funktionalen Verschiedenheiten der Geschlechter rechtfertigen lässt und welche daher gegen Art. 4 Abs. 2 BV verstösst (Erw. II/2).» Die entsprechenden kantonalen Anspruchsvo-

raussetzungen durften im konkreten Fall nicht angewendet werden (Erw. II/3).

12 wer eine Rente der 2. Säule gestützt auf Art. 4 Abs. 2 aBV zugesprochen hatte, wurde der Bundesgesetzgeber tätig. Er hat im Rahmen der 10. AHV-Revision die Witwerrente ins AHVG (1. Säule) aufgenommen. Anspruch auf eine Witwenoder Witwerrente haben Witwen oder Witwer, sofern sie im Zeitpunkt der Verwitwung Kinder haben (Art. 23 Abs. 1 AHVG). In dieser Bestimmung sind Frauen und Männer gleichgestellt. Es existieren jedoch auch besondere Bestimmungen. Nach Art. 24 Abs. 1 AHVG haben Witwen überdies Anspruch auf eine Witwenrente, wenn sie im Zeitpunkt der Verwitwung keine Kinder oder Pflegekinder, jedoch das 45. Altersjahr vollendet haben und mindestens fünf Jahre verheiratet gewesen sind. War die Witwe mehrmals verheiratet, so wird auf die Gesamtdauer der Ehen abgestellt. In Absatz 2 wird festgehalten: Zusätzlich zu den in Artikel 23 Absatz 4 aufgezählten Beendigungsgründen (Tod oder Wiederverheiratung) erlischt der Anspruch auf die Witwerrente, wenn das letzte Kind des Witwers das 18. Altersjahr vollendet hat. ${ }^{18}$

$18 \overline{\text { Der Bundesrat führte in der Botschaft über die }}$ zehnte Revision der Alters- und Hinterlassenenversicherung vom 5. März 1990 (BBl 1990 II 1) S. 37 Folgendes aus: «Die geltende Gesetzgebung kennt nur die Witwen-, nicht aber die Witwerrente. Immer häufiger geht aber heute auch die Ehefrau ganz oder teilweise einer Erwerbstätigkeit nach. In den Fällen, in denen der Mann den Haushalt führt und die Kinder betreut, verfügt er für sich über keinerlei Absicherung der AHV, falls seine Frau verstirbt. Wir schlagen Ihnen daher die Einführung der Witwerrente vor. Der Anspruch soll aber auf die Fälle beschränkt werden, in denen der Witwer Kinder unter 18 Jahren zu versorgen hat. Wir sind uns bewusst, dass mit dieser Einschränkung Witwen- und Witwerrente 
G wurde die Witwerrente später verankert. In der Botschaft zur 1. BVGRevision finden sich folgende Ausführungen: «Das BVG sieht in Artikel 19 nur die Gewährung einer Witwenrente vor. Diese Bestimmung steht im Widerspruch zum Gleichberechtigungsartikel der Bundesverfassung (Art. 4 Abs. 2 aBV; Art. 8 Abs. 3 nBV). (...) Anders als die AHV wird die berufliche Vorsorge eine Witwerrente ausrichten, welche den Bedingungen der Witwenrente voll entspricht.» 19

Im Sozialversicherungsrecht nähert sich der Gesetzgeber der verfassungsmässigen Ordnung bzw. dem Grundsatz der Gleichbehandlung der Geschlechter (Art. 8 Abs. 3 BV) demnach an. Wieso die Ungleichbehandlung von Witwern und Witwen im Bereich der 1. Säule gerechtfertigt sein soll, bleibt freilich offen. Erklärt werden kann die verbleibende Ungleichbehandlung mit der traditionellen Rollenverteilung zwischen Mann und Frau bzw. dem Leitbild der Versorgerehe. ${ }^{20}$

\section{AHV-Rentenalter}

15 Anton Achermann vollendete am 22. August 1976 das 62. Altersjahr. Am 17. Januar 1977 ersuchte er die Ausgleichskasse des Kantons Luzern um Be-

nicht gleich geregelt werden. Wir halten den Unterschied jedoch einstweilen noch für gerechtfertigt.»

19 Botschaft zur Revision des Bundesgesetzes über die berufliche Alters-, Hinterlassenen- und Invalidenvorsorge (BVG) (1. BVG-Revision) vom 1. März 2000 (BBl 2000 2637) S. 2661. Die Anspruchsvoraussetzungen für die Rente finden sich noch heute in Art. 19 BVG.

20 Margrith Bigler-Eggenberger, Der EWR-Vertrag und die Gleichstellung von Frau und Mann in der Schweiz (Arbeits- und Sozialversicherungsrecht), AJP 1992 S. 1347 ff., S. 1357. freiung von der Beitragspflicht sowie um Ausrichtung einer Altersrente, beides rückwirkend auf den 22. August 1976. Nach Art. 21 Abs. 1 AHVG hatten Männer einen Rentenanspruch, nachdem Sie das 65. Altersjahr zurückgelegt haben. Frauen waren dagegen bereits im Alter von 62 anspruchsberechtigt. Herr Achermann berief sich dabei auf Art. 4 BV und die Europäische Konvention zum Schutze der Menschenrechte und Grundfreiheiten (EMRK). Mit Verfügung vom 21. Januar 1977 lehnte die Ausgleichskasse beide Begehren ab, wobei sie darauf hinwies, dass sie an Art. 3 Abs. 1 und Art. 21 Abs. 1 AHVG, welche Beitragspflicht und Rentenanspruch altersmässig umschreiben, gebunden sei. Die dagegen erhobene Beschwerde des Herrn Achermann wies das Verwaltungsgericht des Kantons Luzern ab. Vor Bundesgericht rügte der Beschwerdeführer die Verletzung des Grundsatzes der Gleichbehandlung von Mann und Frau (Art. 4 Abs. 2 aBV; Art. 8 Abs. $3 \mathrm{nBV}$ ) sowie des völkerrechtlichen Diskriminierungsverbotes (Art. 14 EMRK). Betreffend Art. 4 Abs. 2 aBV; Art. 8 Abs. 3 nBV stellte das Bundesgericht fest, dass «die von der Bundesversammlung erlassenen Gesetze (...) vom Richter nicht auf ihre Verfassungsmässigkeit überprüft werden können (Art. 113 Abs. 3 und Art. 114 bis Abs. 3 BV).»21 Zur EMRK-Rüge stellte das Bundesgericht fest: «Die EMRK und die von der Schweiz nur zum Teil ratifizierten - Zusatzprotokolle enthalten weder eine allgemeine Vorschrift über die rechtsgleiche Behandlung von Mann und Frau noch eine im vorliegenden Fall anwendbare Bestimmung über die Altersgrenzen beim Anspruch auf Sozialversicherungsleistungen bzw. bei der Ver-

21 BGE 105 V 1 S. 2; vgl. den heutigen Art. 190 BV. 
pflichtung zu entsprechenden Beiträgen. Art. 3 Abs. 1 und Art. 21 Abs. 1 AHVG stehen demnach nicht in einem Widerspruch zur EMRK, weshalb die Verwaltungsgerichtsbeschwerde auch in diesem Punkt unbegründet ist.» ${ }^{22}$ Anton Achermanns Beschwerde wurde abgewiesen. ${ }^{23}$

Im Rahmen der 10. AHV-Revision hat die Bundesversammlung die Voraussetzungen für die Altersrente angepasst. Das Rentenalter der Frau wurde auf 64 erhöht, das des Mannes ist gleichgeblieben (Art. 21 Abs. 1 AHVG). Zwingende biologische und funktionale Unterschiede für die Rechtfertigung der Ungleichbehandlung der Geschlechter sind hier nicht auszumachen. Ursache - nicht Rechtfertigung - der Ungleichbehandlung ist vielmehr die Tradition. ${ }^{24}$ Die Eidgenössische Volksinitiative «Für eine generationengerechte Altersvorsorge (Vorsorge Ja - aber fair)»25 sowie die Volksinitiative «Für eine sichere und nachhaltige Al-

22 BGE $105 \mathrm{~V} 1 \mathrm{~S} .4$.

23 Siehe ferner BGE $109 \mathrm{Ib} 81$.

24 Vgl. jedoch Bigler-Eggenberger (Fn. 20), 1358: «Die generell schlechtere Stellung der Frauen im Arbeitsleben, ihre häufige Doppelbelastung mit familiären Pflichten, ihre aus familiären Gründen oft unterbrochene Berufskarriere rufen nun aber vorläufig nach einer solchen Ungleichbehandlung in diesem speziellen Bereich für so lange, als ihre effektive Schlechterstellung im gesamten gesellschaftlichen und wirtschaftlichen Bereich andauert, wobei allerdings sich sogleich das Problem stellt, ob damit nicht die traditionelle Rollenverteilung zulasten der Frauen weiterhin zementiert werde.»

25 Die Initiative schlägt Folgendes vor (Vorprüfung der Eidgenössischen Volksinitiative «Für eine generationengerechte Altersvorsorge [Vorsorge Ja aber fair]» vom 19. März 2019 [BBl 2019 2679] S. 2681): «Das für die Verwaltung der Renten notwendige Referenzrücktrittsalter (in der 1. und 2. Säule) wird unter Berücksichtigung der Lebenserwartung regelmässig angepasst. Es ist für Frauen und Männer gleich. Der Zeitpunkt der effektiven Pensionierung wird individuell festgelegt.» tersvorsorge (Renteninitiative)» ${ }^{26}$ versuchen, das Rücktrittsalter von Frau und Mann zu vereinheitlichen. Beide Volksbegehren befinden sich im Sammelstadium. ${ }^{27}$

\section{Wehrpflicht und Ersatzabgaben}

17 A. wurde am 20. Dezember 2005 aus der Rekrutenschule entlassen, nachdem er am 19. Januar 2005 für dienstuntauglich befunden worden war. Mit Verfügung vom 22. Oktober 2015 legte die Wehrpflichtersatzverwaltung des Kantons Zug den Wehrpflichtersatz von A. für das Ersatzjahr 2014, ausgehend von einem taxpflichtigen Einkommen von Fr. 55'400.-, auf Fr. 1'662.- fest, wovon ein bereits bezahlter Betrag von Fr. 600.- in Abzug gebracht wurde. Die dagegen gerichtete Einsprache wurde am 19. November 2015 abgewiesen. A. erhob Beschwerde an das Verwaltungsgericht des Kantons Zug, welches das Rechtsmittel mit Urteil vom 27. September 2016 abwies. Mit Eingabe vom 16. November 2016 erhob A. beim Bundesgericht Beschwerde in öffentlich-rechtlichen Angelegenheiten (eventuell Verfassungsbeschwerde) mit dem Antrag, er sei in Aufhebung des angefochtenen Entscheids von der Wehrpflicht bzw. der Wehrpflichtersatz-

$26 \overline{\text { Das Rentenalter soll danach für beide Geschlech- }}$ ter auf 66 Jahre festgesetzt werden. Periodische Anpassungen des Rentenalters sind möglich, wenn sich die Lebenserwartung der Bevölkerung verändert: Vorprüfung der Eidgenössischen Volksinitiative «Für eine sichere und nachhaltige Altersvorsorge (Renteninitiative)» vom 22. Oktober 2019 (BBl 2019 7204) S. 7207.

27 Übersicht über die hängigen Volksinitiativen im Sammelstadium. Dem Gleichbehandlungsgebot wäre natürlich auch Genüge getan, wenn das Rentenalter der Männer auf das Alter 64 gesenkt würde. Mitunter aufgrund der Finanzierungsproblematik dürfte diese Variante, welche nicht nur grundrechtskonform ist, sondern auch den sozialversicherungsrechtlichen Standard in der Schweiz verbessert, kaum mehrheitsfähig sein. 
abgabe zu befreien. A. rügte die Verletzung des verfassungsrechtlichen und des völkerrechtlichen Diskriminierungsverbots (Art. 8 Abs. 2 und 3 BV, Art. 14 EMRK). Das Bundesgericht pflichtete dem Beschwerdeführer grundsätzlich bei, dass die auf Männer beschränkte Wehrpflicht im Widerspruch zu Art. 8 Abs. 2 und 3 BV steht. Dass biologische und funktionale Unterschiede Frauen grundsätzlich für den Militärdienst untauglich erscheinen lassen, werde dadurch widerlegt, «dass Frauen freiwillig Militärdienst leisten können (Art. 59 Abs. 2 BV; Art. 3 Abs. 1 des Bundesgesetzes vom 3. Februar 1995 über die Armee und die Militärverwaltung [MG; SR 510.10]), dass sie in der Armee grundsätzlich die gleichen Rechte und Pflichten haben wie die männlichen Angehörigen der Armee (Art. 3 Abs. 3 MG) und ihnen alle Funktionen offenstehen. Auch in den meisten ausländischen Armeen werden Frauen in mehr oder weniger grossem Umfang eingesetzt. In einigen Staaten besteht auch eine Wehrpflicht für Frauen.»28 Das Bundesgericht stellte jedoch weiter fest, dass Art. 59 Abs. 2 BV die Wehrpflicht bewusst und gewollt auf Männer beschränke. Art. 59 Abs. 2 BV gehe dem verfassungsrechtlichen Diskriminierungsverbot als «lex specialis» vor. ${ }^{29}$ Ferner statuiere die EMRK kein allgemeines Diskriminierungsverbot. Art. 14 komme nur auf die in der Konvention anerkannten Rechte zur Anwendung. Solche Rechte würden durch die Wehr-

$28 \overline{\text { Urteil des Bundesgerichts 2C 1051/2016 vom }}$ 24. August 2017 E. 3.3. Siehe auch die Urteilsbesprechung: Martin D. Küng/Bernhard Waldmann, Wehrpflichtersatzabgabe: Ungleichbehandlung der Geschlechter, in: Jusletter 23. April 2018.

29 Urteil des Bundesgerichts 2C_1051/2016 vom 24. August 2017 E. 3.4. pflicht nicht betroffen. Die Wehrpflicht sei insbesondere vom Verbot der Sklaverei und Zwangsarbeit ausgenommen (Art. 4 Ziff. 3 lit. b EMRK).30 Die Beschwerde erweise sich damit als unbegründet. ${ }^{11}$

18 Die Auffassung des Bundesgerichts, dass Art. 59 Abs. 2 BV dem Diskriminierungsverbot vorgeht, ist nicht unbestritten. $3^{2}$ Waldmann ist der Ansicht, dass die Geschlechtergleichheit eine Kerngehaltsbestimmung darstelle, die auch den Verfassungsgeber selber binde. 33 Bondolfi geht in die gleiche Richtung. Sie weist sogar darauf hin, dass Art. 190 BV der Korrektur eines verfassungswidrigen Bundesgesetzes nicht entgegenstehe, sofern das Bundesgesetz den Kerngehalt der Grundrechte nicht wahre. 34 Nach der hier vertretenen Ansicht stellt die Wehrpflicht bzw. jede Form von Zwangsarbeit einen Eingriff in den Kerngehalt der Wirtschaftsfreiheit (Art. 27 BV) dar, unabhängig davon, ob die Wehrpflicht sich bloss auf Männer oder auf beide Ge-

30 Urteil des Bundesgerichts 2C_1051/2016 vom 24. August 2017 E. 4.1 f.; vgl. auch Urteil des Bundesgerichts 2C_221/2009 vom 21. Januar 2010 E. 2.1; Urteil des Bundesgerichts 2C_583/2015 vom 12. Februar 2016 E. 2.1; Urteil des Bundesgerichts 2C_396/2012 vom 23. November 2012 E. 2.2 und 3; Urteil des Bundesgerichts 2A.47/2002 vom 23. Mai 2002 E. 2.2; Urteil des Bundesgerichts 2A.433/1990 vom 17. September 1991 E. 3.

31 Urteil des Bundesgerichts 2C_1051/2016 vom 24. August 2017 E. 5.

32 Ulrich Häfelin/Walter Haller/Helen Keller/Daniela Thurnherr, Schweizerisches Bundesstaatsrecht, 9. Aufl., Zürich 2016, S. 229 Rz. 779.

33 Bernhard Waldmann, Das Diskriminierungsverbot von Artikel 8 Absatz 2 BV als besonderer Gleichheitssatz, Bern, S. 620 f.

34 Sibilla Bondolfi, Wehrpflicht und Geschlechterdiskriminierung: Verfassungsrechtliche und völkerrechtliche Anforderungen an die Wehrpflicht im Vergleich zum Modell Israel, Diss., Zürich 2012, S. 277. 
schlechter erstreckt. 35 Es ist jedoch nicht davon auszugehen, dass das Bundesgericht seine Rechtsprechung ändern wird. Es wäre Sache des Volkes, die Grundrechte im Bereich des Militärwesens zur Geltung zu bringen. ${ }^{6}$ Von der Wehrpflicht ist allerdings nur ein kleiner Teil des Volkes betroffen. Jener Teil des Souveräns, der der Pflicht nicht unterliegt, scheint ein grosses Interesse an den Diensten der Pflichtigen zu haben. Die Abschaffung der Dienstpflicht ist entsprechend unpopulär: Die eidgenössische Volksinitiative «Ja zur Aufhebung der Wehrpflicht» der Gruppe für eine Schweiz ohne Armee (GSoA) wurde am 22. September 2013 von 73,2 Prozent der Stimmenden und allen Kantonen abgelehnt.37

Um dem Gleichbehandlungsgebot Genüge zu tun, könnte die Wehrpflicht auch auf Frauen ausgedehnt werden. Da die Wehrpflicht - wie jede Dienstpflicht jedoch eine Form von Zwangsarbeit darstellt und damit den Kerngehalt der Wirtschaftsfreiheit verletzt, würde die

35 «Die Militärdienstpflicht beeinträchtigt die Dienstpflichtigen in der Ausübung ihres Berufes oder in ihrer Ausbildung. Ein Eingriff in die Wirtschaftsfreiheit, welche insbesondere den freien Zugang zu einer privatwirtschaftlichen Erwerbstätigkeit und deren freie Ausübung gewährleistet, ist daher offensichtlich»: Bondolfi (Fn. 34), 189. Vgl. nun auch Martin Kocher, Die bundesgerichtliche Kontrolle von Steuernormen Grundlagen, Anfechtungsobjekt, Streitgegenstand, Verfahren, Fallstudien, Bern 2018, Rz. 1809.

36 Urteil des Bundesgerichts 2C_1051/2016 vom 24. August 2017 E. 3.4.

37 Bundesratsbeschluss vom 18. November 2013 über das Ergebnis der Volksabstimmung vom 22. September 2013 (Volksinitiative «Ja zur Aufhebung der Wehrpflicht»; Bundesgesetz über die Bekämpfung übertragbarer Krankheiten des Menschen (Epidemiengesetz, EpG; SR 818.101); Änderung des Bundesgesetzes über die Arbeit in Industrie, Gewerbe und Handel (Arbeitsgesetz, ArG) (BBl 2013 8789) S. 8793.
Ausdehnung der Wehrpflicht auf Frauen mit Blick auf die Verwirklichung der Grundrechte (Art. 35 Abs. 1 BV) keine Verbesserung des Zustandes bedeuten. Gleichbehandlung alleine ist kein Mass für eine gute Behandlung.

\section{Ein Grenzfall: Der Vergewaltigungs- tatbestand (Art. 190 StGB)}

2o Angriffe auf die sexuelle Freiheit und Ehre von Männern werden im geltenden Recht von Art. 189 Abs. 1 StGB erfasst. Danach wird mit Freiheitsstrafe bis zu zehn Jahren oder Geldstrafe bestraft, wer eine Person zur Duldung einer beischlafsähnlichen oder einer anderen sexuellen Handlung nötigt, namentlich indem er sie bedroht, Gewalt anwendet, sie unter psychischen Druck setzt oder zum Widerstand unfähig macht. Der erzwungene Beischlaf mit Frauen fällt dagegen unter Art. 190 StGB. Nach dieser Norm wird mit Freiheitsstrafe von einem Jahr bis zu zehn Jahren bestraft, wer eine Person weiblichen Geschlechts zur Duldung des Beischlafs nötigt, namentlich indem er sie bedroht, Gewalt anwendet, sie unter psychischen Druck setzt oder zum Widerstand unfähig macht.

Das StGB befindet sich aktuell in der Revision. Nach der Revisionsvorlage lautet Art. 189 Abs. 1 StGB wie folgt: «Wer eine Person zur Vornahme oder Duldung einer sexuellen Handlung nötigt, namentlich indem er sie bedroht, Gewalt anwendet, sie unter psychischen Druck setzt oder zum Widerstand unfähig macht, wird mit Freiheitsstrafe bis zu fünf Jahren bestraft.» Art. 190 Abs. 1 StGB soll neu folgenden Wortlaut haben: «Wer eine Person zur Vornahme oder Duldung des Beischlafs oder einer beischlafsähnli- 
chen Handlung, insbesondere einer solchen, die mit einem Eindringen in den Körper verbunden ist, nötigt, namentlich indem er sie bedroht, Gewalt anwendet, sie unter psychischen Druck setzt oder zum Widerstand unfähig macht, wird mit Freiheitsstrafe von zwei bis zu zehn Jahren bestraft.» 38 Gemäss der Botschaft des Bundesrates sollen auch Männer als Opfer einer Vergewaltigung in Frage kommen und ihr Trauma rechtlich anerkannt werden. 39

Gebietet die Verfassung bzw. Art. 8 Abs. 3 BV die gleiche Beurteilung von Straftaten gegenüber männlichen und weiblichen Opfern sexueller Handlungen bzw. ist in deren ungleichen Behandlung eine Diskriminierung zu erblicken? Niggli/Maeder weisen darauf hin, dass Frauen aufgrund einer Vergewaltigung schwanger werden können.40 Entsprechend votieren die Autoren für die Beibehaltung der ungleichen Beurteilung von Straftaten gegenüber männlichen und weiblichen Opfern: «Für eine Revision des Vergewaltigungstatbestandes (Art. 190 StGB) bzw. des Tatbestandes der sexuellen Nötigung (Art. 189 StGB) im diskutierten politischen Sinne besteht kein ersichtlicher Bedarf. Die Unterscheidung in Beischlaf/beischlafsähnlich stellt (...) nicht auf das Geschlecht ab, sondern auf die Möglichkeit einer Schwangerschaft bzw. der Fortpflanzung. Die Unterscheidung ist weder veraltet noch obsolet. Dies zeigt die aktuelle

38 Bundesgesetz über die Harmonisierung der Strafrahmen (Entwurf) BBl $20182967 \mathrm{ff}$.

39 Botschaft vom 25. April 2018 zur Harmonisierung der Strafrahmen und zur Anpassung des Nebenstrafrechts an das geänderte Sanktionenrecht (BBl 2018 2827) S. 2873 f.

40 Siehe Marcel Alexander Niggli/Stefan Maeder, Beischlaf, parlamentarische Vorstösse und andere erregende Dinge, AJP 2016 S. 1159 ff., S. 1175.
Rechtslage, die z.B. Ehe und eingetragene Partnerschaft unterscheidet. Abgesehen von der Bezeichnung würden die Vorschläge weder Umfang noch Anwendungsbereich noch Mass der heute geltenden Strafbarkeit verändern. Eine Revision aber, die einzig eine Änderung der Bezeichnung anstrebt und deren Motiv zudem auf fundamentalen Missverständnissen des geltenden Rechts beruht, ist weder sinnvoll noch nötig, angesichts des medientauglichen Gegenstandes aber hoch gefährlich.»41

23 Nach der hier vertretenen Auffassung stellt die Unterscheidung Beischlaf/ beischlafsähnlich in den Art. 189 f. StGB nicht einzig auf die Möglichkeit einer Schwangerschaft ab, da auch der erzwungene Beischlaf mit Frauen ausserhalb des gebärfähigen Alters unter Art. 190 StGB fällt. Die ungleiche Beurteilung von Straftaten gegenüber gebärfähigen Frauen und den restlichen Menschen liesse sich mit biologischen Unterschieden rechtfertigen. Wenn im Anwendungsbereich von Art. $189 \mathrm{f}$. StGB tatsächlich opferbezogene Differenzierungen angezeigt sein sollten, müsste ein spezieller Tatbestand für den Schutz gebärfähiger Frauen vor sexuellen Übergriffen geschaffen werden. Geradezu geboten erscheint eine solche Differenzierung auf der Tatbestandsebene jedoch nicht. Wenn der Übergriff für ein Opfer besonders gravierende Folgen hat (z.B. eine ungewollte Schwangerschaft), kann dem im Rahmen der Strafzumessung Rechnung getragen werden (Art. 47 Abs. 2 StGB). Mit Blick auf Art. 8 Abs. 3 BV ist die geplante Revision des Strafrechts jedenfalls zu begrüssen.

41 Niggli/Maeder (Fn. 40), S. 1175. 


\section{Schlussfolgerungen}

24 «Die gesellschaftlich eingespielte Rollenverteilung und die damit verbundenen Stereotypen sind (immer noch) eine wichtige Ursache abwertender Differenzierungen zu Lasten der Frauen (...). Dazu kommen verschiedenste weitere Ursachen einer tatsächlichen Schlechterstellung von Frauen in Gesellschaft, Wirtschaft und Staat.»42 - Frauen mögen in verschiedenen Bereichen de facto schlechter gestellt sein. Offensichtlich ist jedoch, dass Männer in verschiedenen Bundesgesetzen benachteiligt bzw. Frauen privilegiert werden. Eine Ungleichbehandlung der Geschlechter kann im Sexualstrafrecht gerechtfertigt sein (vorstehend IV). Im Sozialversicherungsrecht (vorstehend III 1 und 2) ist die Ungleichbehandlung nicht gerechtfertigt, sondern lediglich durch die traditionelle Rollenverteilung zwischen Mann und Frau erklärbar. Im Militärwesen gilt das Gleiche (vorstehend III 3).

Die Möglichkeiten der Verwaltungsbehörden und Gerichte zur Korrektur grundrechtswidriger Bundesgesetze sind eingeschränkt: Nach Art. 190 BV sind Bundesgesetze - auch verfassungswidrige - für die rechtsanwendenden Behörden verbindlich. Es bleibt daher Sache des Gesetzgebers, mittels Gesetzesrevisionen darauf hinzuwirken, dass die Grundrechte in der ganzen Rechtsordnung zur Geltung kommen (Art. 35 Abs. 1 BV). Mit Blick auf Art. 8 Abs. 3 BV sollte der Gesetzgeber darauf achten, beide Geschlechter gleich gut und nicht gleich schlecht zu stellen. Das zeigt die Entwicklung im Namensrecht: Durch die Streichung der Doppelnamen bei Ehegat- ten ist die Geschlechterdiskriminierung im Namensrecht zwar beseitigt, gleichzeitig werden jedoch die Möglichkeiten der Brautleute bei der Namenswahl beschnitten. Jüngst ist wieder der Ruf nach der Einführung der Doppelnamen laut geworden (oben II).

42 SGK BV-Schweizer (Fn. 1), Art. 8 N 69. 